\title{
Títere en la modalidad virtual: Recurso didáctico interactivo para nivel inicial
}

\author{
Puppets in the virtual modality: interactive teaching resource for early \\ childhood education
}

1 Daniela Benalcázar Chicaiza

https://orcid.org/0000-0003-1990-634X

Universidad Técnica de Ambato, Facultad de Ciencias Humanas y de la Educación. Ambato, Ecuador.

da.benalcazar@uta.edu.ec

2 Mayra Isabel Barrera Gutiérrez

https://orcid.org/0000-0002-3550-7173

Universidad Técnica de Ambato, Facultad de Ciencias Humanas y de la Educación.

Ambato, Ecuador.

mayraibarrerag@uta.edu.ec

3 Mery Fernanda Pozo Ruiz

https://orcid.org/0000-0003-1920-0080

Universidad Técnica de Ambato, Facultad de Ciencias Humanas y de la Educación.

Ambato, Ecuador.

mpozo6571@uta.edu.ec

4 Carolina Elizabeth San Lucas Solórzano (iD) https://orcid.org/0000-0001-9791-3110.

Universidad Técnica de Ambato, Facultad de Ciencias Humanas y de la Educación.

Ambato, Ecuador.

carolinaesanlucass@uta.edu.ec

Artículo de Investigación Científica y Tecnológica

Enviado: 24/12/2021

Revisado: 29/12/2021

Aceptado: $12 / 01 / 2022$

Publicado:08/03/2023

DOI: https://doi.org/10.33262/concienciadigital.v6i1.4.1989

Cítese:

Benalcázar Chicaiza, D., Barrera Gutiérrez, M. I., Pozo Ruiz, M. F., \& San Lucas Solórzano, C. E. (2023). Títere en la modalidad virtual: Recurso didáctico interactivo para nivel inicial. ConcienciaDigital, 6(1.4), 109-122. https://doi.org/10.33262/concienciadigital.v6i1.4.1989

CONCIENCIA DIGITAL, es una Revista Multidisciplinar, Trimestral, que se publicará en soporte electrónico tiene como misión contribuir a la formación de profesionales competentes con visión humanística y crítica que sean capaces de exponer sus resultados investigativos y científicos en la misma medida que se promueva mediante su intervención cambios positivos en la sociedad. https://concienciadigital.org _

La revista es editada por la Editorial Ciencia Digital (Editorial de prestigio registrada en la Cámara Ecuatoriana de Libro con No de Afiliación 663) www.celibro.org.ec 


\section{Palabras}

claves: títere, recurso

didáctico interactivo, interacción, educación inicial, modalidad virtual.

Keywords:

puppet, interactive teaching resource, interaction, early childhood education, virtual

\section{Resumen}

Introducción. La educación virtual es una realidad inevitable en la actualidad tras los efectos de la pandemia a nivel mundial. Muchos sectores se han visto afectados entre ellos la educación infantil, sin duda alguna los docentes han tenido que adaptar metodologías, estrategias y recursos para poder llegar hacia los estudiantes y continuar con la escolaridad desde casa. Objetivo: Analizar al títere como un recurso didáctico interactivo que ayuda en la modalidad virtual para el proceso de aprendizaje en el Nivel Inicial. Metodología: La metodología que se empleó se basó en la teoría crítica, con diseño emergente, de enfoque cualitativo, de alcance descriptivo y explicativo con el que se cumplieron con los objetivos planteados al usar las técnicas entrevista y observación con sus respectivos instrumentos. Resultados. Entre los resultados más relevantes que se obtuvieron al analizar la información obtenida, se argumenta que los títeres son usados para crear una relación de confianza dentro de la sala virtual en el presente contexto educativo; hoy en día se ha visto la necesidad de mejorar el ambiente de aprendizaje y convertirlo en un espacio de juego trabajo a pesar de la virtualidad. Conclusión. Tras el análisis de la información recolectada, se arribó a la conclusión de que el títere, es un recurso didáctico interactivo, que entre sus múltiples objetivos potencia las relaciones interpersonales desde un ambiente adecuado. Como recurso el títere, ofrece un sinnúmero de ventajas en el proceso de aprendizaje, como son la concentración, estimulación de la imaginación, desarrollo de habilidades sociales y comunicativas, la oportunidad de expresión, desarrollo de la espontaneidad y la reflexión

\section{Abstract}

Introduction. Virtual education is an inescapable reality today. After the effects of the global pandemic, many sectors have been affected, including early childhood education. Undoubtedly, teachers have had to adapt methodologies, strategies and resources to be able to reach students and continue with schooling from home. Objective: Analyze puppets as an interactive teaching resource that helps in the virtual modality for the classroom learning process at the early childhood level. Methodology: The methodology used was based on critical theory with an emergent design, qualitative 
modality. approach and a descriptive and explanatory scope. The established objectives were achieved using interview and observation techniques with their respective instruments. Results. Among the most relevant results obtained when analyzing the information gathered, it is argued that puppets are used to create a relationship of trust within the virtual classroom in the present educational context; at present the need to improve the learning environment and turn it into a work-play space, despite its virtuality, is clear. Conclusion. After analyzing the information collected, it was concluded that the puppet is an interactive didactic resource which, among its multiple objectives, enhances interpersonal relationships from a suitable environment. As a resource, puppets offer countless advantages in the learning process such as concentration, stimulation of the imagination, development of social and communication skills, the opportunity for expression, development of spontaneity, and reflection.

\section{Introducción}

El aprendizaje a nivel mundial se ha visto afectado por la pandemia del COVID 19, sin embargo, se han empleado numerosos recursos para captar la atención del niño especialmente en la Educación Inicial, uno de estos es el títere que teniendo en cuenta la importancia y la contribución que generan las emociones en el proceso de aprendizaje lejos de ser un complemento es una forma de aprendizaje, así el títere en el aula genera un sinfín de emociones que provocan aprendizajes (Ramírez, 2019).

El títere pensado como acción poética, fenómeno que trasciende la realización plástica del objeto que lo encarna (Martínez, 2017), es un agente mediador "entre" lo que el docente desea alcanzar, los objetivos que se propone y las necesidades de sus niños. En Latinoamérica son numerosos los recursos a los cuales se han visto abocados los docentes a usar para poder generar aprendizajes reales con los niños en la modalidad virtual, y claro está que se ha considerado al títere como uno de ellos (Salazar, 2019).

Así mismo en Ecuador los docentes han sufrido cambios abruptos en las metodologías y estrategias que solían emplear debido a la modalidad virtual a la que han tenido que enfrentarse por la pandemia que asecha al mundo, se debe comprender que el desarrollo integral del niño es un factor esencial que debe atenderse con especial cuidado, en el Nivel Inicial los niños desarrollan todas sus habilidades de forma espiral, por lo que es necesario que las destrezas sean adquiridas a través de juegos y mediante la interrelación del niño con su entorno y pares sociales (Salto \& Cendón, 2019), un medio para que estas destrezas 
sean adquiridas especialmente en modalidad virtual es el títere que se lo ha tomado como recurso interactivo en el nivel inicial (Szulkin \& Correa, 2019).

Para comprender la importancia del uso del títere es necesario partir desde una perspectiva general comprendiendo que es un recurso en el Nivel Inicial, al igual que el significado del recurso didáctico interactivo, y como estos se vinculan con el títere para mejorar la interacción dentro de las horas sincrónicas en la modalidad virtual, al igual que percibir el valor que tiene dentro de la praxis y como secunda en el proceso de enseñanza aprendizaje.

Se entiende que, una metodología en el nivel inicial es un proceso sistematizado que promueve el desarrollo integral de los educandos, es por ello por lo que el Ministerio de Educación establece el Currículo de Educación Inicial como la guía en la cual se evidencia la metodología de juego trabajo, considerando al juego como una actividad innata y significativa en la infancia. Por otro lado, la Guía Metodológica es conocida como la metodología que trabaja por rincones, la que consiste en la organización del aula en distintos espacios de aprendizaje, esta metodología optimiza la labor docente, permitiendo que niños tengan un desarrollo integral continuo y significativo promueven múltiples aprendizajes, potenciando las capacidades e intereses de los párvulos (Ministerio de Educación, 2014).

Un estudio realizado por Reid-Searl et al. (2017), expone que un aprendizaje significativo se da gracias al juego ya que, a través de actividades experimentales específicas y orientadas a las necesidades de un desarrollo y enfocadas en el proceso de aprendizaje de los infantes puede sembrar en ellos los conocimientos necesarios que le permitan asimilar su contexto. Sumado a lo antes mencionado la metodología aplicada en el Nivel Inicial es significativa para los infantes y su desarrollo.

Por otra parte teorizar el uso del títere como un recurso didáctico interactivo, obliga a brindar una perspectiva del mismo en la actualidad, al pasar la Educación Inicial de una modalidad presencial a una virtual, la dinámica de las clases se desarrolla bajo las llamadas horas sincrónicas y asincrónicas que se abordarán más adelante en el presente texto, sin embargo, las horas sincrónicas que en un principio se las concibe como el tiempo real que el docente acompaña al estudiante, se convierten en todo un desafío, en búsqueda de atención, colaboración y participación activa de los niños de 3 a 5 años, así, uno de los recursos que toman protagonismo para esta edad es el títere.

Es preciso definir al recurso didáctico como un medio de apoyo en la labor docente, que permite la transmisión de información de una manera simple hacia su alumno (Herrera, 2017). Del mismo modo los recursos didácticos interactivos contribuyen en la labor docente, además mejoran la relación y comunicación docente-alumno y entre pares, favoreciendo la comprensión de contenidos, facilitando la expresión de pensamientos, 
sentimientos y emociones creando un ambiente rico de estímulos que generan aprendizaje (Chancusig et al., 2017).

El títere de cualquier tipo sea este un objeto, muñeco, peluche, guante inflado, calcetines, una caja de cartón, adecuado acorde a las necesidades de las personas, o de la forma en que se lo use, ya que es manejado por un actor o individuo de forma intencional, es importante resaltar que dentro del área educativa no se le ha dado el valor que merece debido al desconocimiento de la técnica y de los beneficios en el mundo infantil (Álvarez \& Hechenleitner, 2019).

Cabe mencionar que Trueba \& Rodríguez de la Flor (2014), encajan al títere dentro de un paradigma interactivo, el cual percibe al niño como un ser independiente, inteligente, autónomo, capaz de desarrollar sus capacidades a través de la interacción con los demás en un entorno que le brinde pasión por aprender (p. 17), siendo el docente un puente entre la realidad y la fantasía dando vida a estos mágicos recursos brindando un ambiente estimulante (Ortiz et al., 2020).

Ahora bien, el títere es considerado una subcategoría del teatro que, en la antigüedad tomó lugar en funciones formativas religiosas, representando a la sociedad, su cultura, costumbres y tradiciones en diversos países del mundo (Zeinali, 2017). Por otra parte, María Signorelli y Mane Bernardo (1962) (como se citó en Albiach, 2013), dan una visión del uso del títere adentrándolo al ámbito educativo, denotándolo como fuente de posibilidades gracias a las multidisciplinar que va formando en los estudiantes.

Los títeres pueden ser usados en cualquier momento de la clase ya sea al inicio, en el desarrollo o cierre siempre cuando esté planificado y haya un correcto uso de este, es decir, en el momento adecuado para que los menores puedan discernir e interiorizar el contenido del momento, para Jurkowski (2013), en su Libro titulado "Aspects of Puppet theatre" para que el títere cumpla con el propósito establecido se lo puede usar previo a una planificación consiente sin importar el momento.

Por ello, el títere es un recurso transcendental dentro de la educación ya que desde la perspectiva de Palomas (2012), ha sido por años un instrumento que ayuda a la expresión de pensamientos, emociones y sentimientos, siendo un medio por el cual él educador puede estimular al niño a participar en clases (p. 4), motivarlo e incluso potencializar la dimensión relacional al permitir esta interacción docente alumno y entre pares (Velazco, 2016), abriendo paso a un diálogo fructífero donde cada uno es capaz de exteriorizar sus respuestas sin limitantes mucho más cuando los títeres se encuentran inmersos (Maridueña, 2019).

Para tener una visión más completa del párrafo anterior, el diccionario de la Real Academia Española (2006), define al pensamiento como el conjunto de ideas de una o 
varias personas para formar un juicio de valor tras una reflexión previa, si bien es cierto existen varios tipos de pensamientos tales como divergente, convergente, creativo, reflexivo, crítico, etc. el uso títere ayuda al desarrollo del pensamiento crítico y reflexivo (Wafunga et al., 2018), para generar un pensamiento crítico es necesario reflexionar, es decir, observar analizar, razonar para establecer un punto de vista, por ello los niños tras el uso del títere realizan este proceso al emitir su opinión sobre diferentes temas propuestos (Gómez \& Botero, 2020).

Las emociones a modo de conmociones producidas por un estímulo pueden ser alegría, tristeza, enojo, asombro (Kaplan, 2018), mientras que los sentimientos son entendidos como la suma de la emoción más el pensamiento debido a la interpretación de factores internos y externos, estos pueden ser amor, solidaridad, respeto, entre otros (p. 17). Se ve como el títere ayuda al desarrollo del pensamiento crítico- reflexivo, y a la expresión de emociones y sentimientos que pueden revelarse en clases tras el uso de este recurso (Pillarés, 2011), hay que dejar en claro que la o el docente es el que brinda esta oportunidad de expresión dentro del ambiente de aprendizaje ya sea este de forma presencial o virtual durante las horas sincrónicas.

Los expertos en el tema enfatizan que los pequeños al inmiscuirse en el ámbito educacional presentan cierto grado de dificultades que limita su comunicación y más cuando se trata de exponer emociones o sentimientos, por ello Raihan Wan Ramli (2012), recomiendan que es necesario incluir historias, muñecos y juegos en la praxis para conectarse con los infantes, de esta manera el títere ayuda a que los niños puedan abrirse al mundo y expresarse sin temor, que al ser de otro modo permanecería oculto.

Por consiguiente, el uso del títere promueve beneficios al infante, permitiéndole comunicarse, sacar a flote sus emociones y sentimientos expresándolos libremente, además aflora su creatividad e imaginación, promoviendo el desarrollo del lenguaje e incluso ayudando a la resolución de conflictos por medio de la manipulación de este (Çağda \& Kalmış, 2015). También ayuda a generar un ambiente cálido en clases, favoreciendo el estado emocional de docentes y alumnos, fortaleciendo su relación y motivación permitiendo que aprendan uno del otro (Kroflin, 2015).

Otros beneficios que se rescatan Mármol (2019), con el uso del títere es que, ayuda a los menores a escapar de la realidad y transportarse a un mundo imaginario. Es muy probable que con el uso de marionetas los infantes logren dar solución a sus conflictos emocionales, a trabajar en equipo, promover diálogos y ganar confianza en sí mismo y en los demás ayudando no solo a que niños puedan sentirse cómodos con ellos mismos si no también con su entorno.

Si bien es cierto el títere es un objeto inanimado, pero por años hemos visto en la televisión ecuatoriana por ejemplo en el programa "veo veo" a su protagonista el perro 
llamado "babau", o en el programa Plaza Sésamo diferentes personajes, los mismos que necesitan de uno o varios individuos que manejen sus bocas, que mueva sus manos o que simplemente mueva sus dedos para darles vida, lo que indudablemente nos ha hecho creer que tiene vida.

Para beneficio de los y las docentes (Rojas, 2007), existen varios tipos de títeres entre los cuales tenemos: el de sombras, hilo o marioneta, el de guante, el de calcetín, el de dedo, con varillas, muppet o bocón, el títere plano, entre otros que permiten sacar el lado artístico dependiendo de la habilidad de cada pedagogo (p. 57), los títeres más adecuados para la educación son los de paleta, los de calcetín y el guiñol o guante (Albiach, 2014).

En cuanto a su inmersión en la modalidad virtual en la actualidad se da gracias a la necesidad de relacionarse entre los miembros de la clase, por lo que se introdujo el títere en horas sincrónicas en las que la interacción entre docente y alumnos se produce con un intercambio de información en tiempo real de forma virtual, mientras que las horas asincrónicas son las enseñanzas que se dan fuera de la sala de trabajo.

En la actualidad docentes de varias instituciones dentro y fuera país han decidido trabajar con títeres no solo para elevar el nivel de atención, sino también para generar un lazo entre docente alumno, en donde el exteriorizar lo que se piensa y se siente durante las clases es importante. Reyes (2020), da a conocer la opinión de docentes que dicen no ser titiriteros pero que este recurso ha sido de gran ayuda para el desarrollo de sus clases y más que un recurso se ha vuelto un compañero de trabajo.

Es por todo lo mencionado que se considera al títere como un recurso didáctico interactivo capaz de mejorar la relación o la misma forma de interactuar entre los miembros de una clase, fortaleciendo su desempeño, mejorado su lenguaje y sobre todo aprendiendo de una manera diferente, interiorizando todo aquello que sienten, piensan, por medio de este el menor es capaz de formarse de manera integral, alcanzando metas a corto o largo plazo propuestas por el educador en la modalidad virtual.

\section{Metodología}

De acuerdo con la naturaleza del estudio, la investigación cuenta con un enfoque cualitativo, debido a que busca comprender el problema planteado con relación al uso inadecuado del títere como recurso didáctico interactivo en las horas sincrónicas de la modalidad virtual del nivel inicial. El cual ha dado paso al tipo de investigación básica, que alude a la producción de teorías o conocimiento (Sampieri et al., 2014).

Además, posee un diseño emergente debido a que la teoría surge de los datos que se han recogido en el en el transcurso de la investigación en libros, revistas científicas y a través de la experiencia. Se establecen dos tipos de alcance, el descriptivo ya que busca detallar la realidad que sucede en las aulas tras el uso del títere como un recurso didáctico que 
apoya a la interacción y explicativo porque pretende establecer las ventajas y desventajas del uso de títeres como recurso interactivo en horas sincrónicas en la modalidad virtual.

Para la recolección de datos se utilizó la técnica de la entrevista con su respectivo instrumento un guion de preguntas y la técnica de la observación, usando como instrumento una guía de observación. Estos instrumentos, fueron elaborados según las categorías provenientes de la sistematización de la variable.

La entrevista constó de 13 preguntas concisas respecto al uso del títere durante las horas clase (sincrónicas) del nivel inicial, interrogantes que fueron validadas por 3 expertos en el área de educación inicial. La validación permitió el ajuste y/o reformulación de las preguntas para ser aplica a 5 educadoras del nivel inicial de la "Unidad Educativa Luis A. Martínez.

La ficha de observación por otro lado se estructuró con 12 ítems a observar los que fueron extraídos de la operacionalización de variables acorde a las preguntas de la entrevista, ficha que fue elaborada tras la aprobación de la entrevista, para proceder a la recolección de información necesaria durante las clases y contrastarlo con la realidad observable del trabajo durante las horas sincrónicas y las respuestas de las entrevistas.

Ambos instrumentos se elaboraron y aplicaron con el fin de recolectar información fehaciente del uso del títere como recurso didáctico interactivo en horas sincrónicas en la modalidad virtual en tiempos de confinamiento. Estas técnicas e instrumentos se escogieron con el fin de que pueda responderán a la pregunta de investigación ¿cómo el títere es utilizado para mejorar la interacción maestra - niños del nivel inicial en horas sincrónicas durante la modalidad virtual?

Esta investigación se realizó en la "Unidad Educativa Luis A. Martínez" cuya población la constituyen docentes y niños del nivel inicial; se contó con el apoyo voluntario y la firma correspondiente de cartas de consentimiento para el proceso de recolección de información.

\section{Resultados}

Se procedió con el análisis en función a las categorías planteadas en la categorización de variables. Categorías que permitieron profundizar en el tema y sistematizar la información recolectada, con este último fin se efectuó una triangulación sobre la información recolectada: entrevistas, ficha de observación y diferentes posturas teóricas abordadas en los anteriores apartados. Este análisis por categorías se evidenció en un cuadro de contraste de la información recolectada. Por la extensión del cuadro mencionado, a continuación, se muestra la estructura de este sin el contenido, pero del cual se hará referencia en posteriores párrafos. 
Tabla 1

Triangulación de información

\begin{tabular}{|c|c|c|c|c|c|}
\hline \multicolumn{5}{|c|}{ Análisis de la Variable: El títere cómo recurso didáctico interactivo } \\
\hline & & & & \\
\hline CATEGORÍAS & & & & \\
\hline & & & & & \\
\hline $\begin{array}{c}\text { Recurso } \\
\text { Didáctico } \\
\text { Interactivo }\end{array}$ & & & & & \\
\hline $\begin{array}{c}\text { Tipos de } \\
\text { títeres }\end{array}$ & & & & & \\
\hline $\begin{array}{c}\text { Interacción } \\
\text { relacional }\end{array}$ & & & & & \\
\hline $\begin{array}{c}\text { Participación } \\
\text { en clases }\end{array}$ & & & & & \\
\hline $\begin{array}{c}\text { Educación } \\
\text { modalidad } \\
\text { virtual }\end{array}$ & & & & & \\
\hline
\end{tabular}

Fuente: Entrevista aplicada a educadoras del Nivel Inicial UE Luis A. Martínez

Como se puede observar en la tabla 1, se procedió con el análisis de El títere cómo recurso didáctico interactivo, en base a 5 categorías, contrastando las respuestas obtenidas en los instrumentos de recolección de información y el argumento teórico para finalmente llegar a resultados de las categorías y de forma general de la variable analizada.

Así, a través de los resultados se argumenta que los títeres son usados para crear una relación de confianza dentro de la sala virtual en el presente contexto educativo, si bien es cierto ha sido usado por años en las clases presenciales, sin embargo, hoy en día se ha visto la necesidad de mejorar el ambiente de aprendizaje y convertirlo en un espacio de juego trabajo como comúnmente es.

Por ello, y con la interrogante que se generó en cuanto a ¿cómo el títere es utilizado para mejorar la interacción docente-alumno en horas sincrónicas de la modalidad virtual del nivel inicial?, y al hacer referencia a autores como Albiach (2014), entre otros, se puede aseverar que el títere es un recurso capaz de entablar una conexión maestra - niños y por lo tanto mejorar la interacción durante las clases virtuales en horas sincrónicas, puesto que, los títeres aunque son objetos inanimados, son divertidos y se usan para la exteriorización de pensamientos, sentimientos y emociones, desarrollando una comunicación con lenguaje claro, comprensible, y dentro de un ambiente de libertad creativa. 


\section{Conclusiones}

- Al ser el títere un objeto inanimado, es la habilidad del docente la que da vida a este recurso en el ámbito educativo; el sustento teórico entorno al uso del títere permite unificar criterios de autores quienes coinciden que este es un recurso didáctico interactivo, que entre sus múltiples objetivos están la potenciación de relaciones interpersonales y desde un ambiente adecuado para niños, niñas y docentes se viabiliza la comunicación lo que dan sentido a la referencia de Vygotsky "los seres humanos somos seres sociales por tal motivo aprendemos de nuestras experiencias al relacionarnos con los otros y con el entorno".

- El uso del títere deja un sinnúmero de ventajas en el proceso de aprendizaje, como lo son la concentración de los niños y niñas, estimulación de la imaginación, el desarrollo de habilidades sociales y comunicativas, la oportunidad de expresión, desarrollo de la espontaneidad y la reflexión, todo esto gracias a una observación consiente y participación activa durante las clases; por otro lado, las desventajas que se presenta giran en torno al tiempo para su elaboración, desarrollo de la aptitud y predisposición de docentes para que su utilización cumpla con el objetivo.

- Dentro del tiempo de clases virtuales que hoy por hoy se han vivido de manera obligatoria por las circunstancias COVID 19, el uso del títere durante las horas sincrónicas tiene más ventajas que desventajas, mismas que fueron develadas y relacionadas con las experiencias de docentes, y que a la final reflejan que el uso del títere contribuye con el desarrollo de la creatividad de los niños, ayuda en la motivación, al desarrollo de la atención, la liberación de pensamientos, sentimientos y emociones creando una relación de confianza con el docente y compañeros, pues se genera un espacio adecuado de comunicación, reflexión y aprendizaje. No menos cierto es la visualización de varias dificultades con el uso del títere, ya que para manejarlos se requiere de elocuencia y habilidad docente desde la virtualidad, a más de lo antes mencionado en cuanto al tiempo para elaborarlos y contextualizarlos a un escenario diferente a la presencialidad, para que este no llegue a caer en la rutina y repetición.

\section{Referencias bibliográficas}

Albiach Oltra, M. À. (2013). Los títeres: un recurso educativo. Educación social. Revista de Intervención Socioeducativa. Obtenido de file://C:/Users/Dell/Downloads/267203-Text\%20de\%201'article-385843-1-10$20141013 \% 20(5) . p d f$

Albiach Oltra, M. A. (2014). El títere como objeto educativo: propuestas de definición y tipologías. Espacios en blanco. Serie indagaciones. Obtenido de http://www.scielo.org.ar/scielo.php?script=sci_arttext\&pid=S1515- 
$94852014000100004 \&$ lang $=\mathrm{pt}$

Álvarez Mabán, E. M. \& Hechenleitner Carvallo, M. I. (2019). Títeres: sus usos en salud y enseñanza en Enfermería. Educación Médica. https://www.sciencedirect.com/science/article/pii/S1575181318301372?via\%3D ihu b

Çă̆da, K. Ç. \& Kalmış, A. (2015). The Role of Puppets in Kindergarten. Open Access Library Journal. https://www.scirp.org/pdf/OALibJ_2016071810353844.pdf

Chancusig, J. C., Flores, G. A., Venegas, G. S. \& Izurieta, E. M. (2017). Utilización de recursos didácticos interactivos a través de las TICS en el proceso de enseñanza aprendizaje en el área de matemática. Boletín Virtual. file:///C:/Users/Dell/Downloads/Dialnet-

UtilizacionDeRecursosDidacticosInteractivosATraves-6119349\%20(1).pdf

Gómez, Gómez, M. P. \& Botero Bedoya, S. M. (2020). Apreciación del docente para contribuir al desarrollo del pensamiento crítico. Eleuthera. https://go.gale.com/ps/retrieve.do?tabID=T002\&resultListType=RESULT_LIST \&s

earchResultsType=SingleTab\&hitCount=2862\&searchType=BasicSearchForm \&cur rentPosition=2\&docId=GALE\% 7CA641749250\&docType=Article\&sort=Relev anc e\&contentSegment=ZONE-Exclude-FT\&prodId=GP

Herrera, Y. (2017). Recursos didácticos para la comprensión de significados por los educandos con retraso. Varona. https://www.redalyc.org/pdf/3606/360657469025.pdf

Jurkowski, H. (2013). Aspects of Puppet Theatre. Reino Unido. https://books.google.com.ec/books?id=o0cdBQAAQBAJ\&printsec=frontcover\& $\mathrm{hl}=\mathrm{es} \&$ source $=\mathrm{gbs} \_\mathrm{ge} \_$summary_r$\& \mathrm{cad}=0 \# \mathrm{v}=$ onepage $\& \mathrm{q} \& \mathrm{f}=$ false

Kaplan, C. (2018). Emociones, sentimientos y afectos: las marcas subjetivas de la educación. Miño y Dávila. https://elibro.net/es/ereader/uta/123449

Kroflin, L. (2015). El poder de los títeres. https://www.unima.org/wpcontent/uploads/2016/10/El_poder_de_la_Marioneta.pdf

Maridueña, R. A. (2019). Teatro de títeres como estrategia para potenciar la atención de niños de 5 años de la Escuela de Educación Básica Isabel Herrera de Velázquez. Conrado. http://scielo.sld.cu/scielo.php?script=sci_arttext\&pid=S1990$86442019000500370 \&$ lang $=\mathrm{pt}$ 
Mármol Maridueña, R. A. (2019). Teatro de títeres como estrategia para potenciar la atención de niños de 5 años de la Escuela de Educación Básica Isabel Herrera De Velázquez. Revista Conrado. http://scielo.sld.cu/pdf/rc/v15n70/1990- 8644-rc15-70-370.pdf

Martínez, G. (2017). Títeres y educación: Manos a la obra (parte II). Trayectoria. Prácticas en Educación artística, 16-25.

Ministerio de Educación. (2014). Currículo de Educación Inicial. Obtenido de Ministerio de Educación: https://educacion.gob.ec/wpcontent/uploads/downloads/2014/06/curriculo-educacion-inicial-lowres.pdf

Ortiz, $_{2}$ B., Bustos Bianchi, M., Canosa, C. \& Santa Cruz, E. (2020). Lenguajes artísticoexpresivos en diálogo: literatura, cine, teatro y títeres. https://elibro.net/es/ereader/uta/129649?prev=as

Palomas, S. (2012). Estrategias metodológicas para la promoción de la salud comunitaria: los títeres tienen la palabra. Argentina: Espacio Editorial. https://elibro.net/es/ereader/uta/66942?as_all=el\%20títere\%20\&as_all_op=unacc ent_icontains\&prev $=$ as\&page $=33$

Pillarés, M. (2011). Emociones y sentimientos: dónde se forman y cómo se transforman. Barcelona: Marge Books. https://elibro.net/es/ereader/uta/41930?as_all=Guia\%20de\%20acción\%20docent e.

icontains\&as_themes=Educación\&as_themes_op=icontains\&

fs_page $=4 \&$ prev $=$ as

Raihan Wan Ramli, W. N. (2012). The Contribution of Shadows Puppet's Show through Engaging Social Communication in Modern Society. Procedia - Social and Behavioral Sciences. https://cyberleninka.org/article/n/548502/viewer

Ramírez Paredes, K.G. (Coord.). (2019). Recursos educativos para el aula del siglo XXI. Eindhoven, NL: Adaya Press. https://www.adayapress.com/wpcontent/uploads/2019/09/RecursosS21.pd

Real Academia Española. (2006). Diccionario de la lengua española. Obtenido de https://www.rae.es/desen/pensamiento

Reid-Searl, K., O'Neill, B., Dwyer, T. \& Crowley, K. (2017). Using a Procedural Puppet to Teach Pediatric Nursing Procedures. Clinical Simulation in Nursing. https://www.nursingsimulation.org/article/S1876-1399(16)301451/fulltext\#secsectitle0035 
Reyes, I. (27 de julio de 2020). El proyecto de títeres en cuarentena para la enseñanza de música. Prensa Libre, Periódico Líder de Guatemala. https://www.prensalibre.com/vida/escenario/el-proyecto-de-titeres-encuarentena- para-la-ensenanza-de-musica/

Rojas, P. (2007). La expresión corporal: una asignatura apasionante. Wanceulen Editorial.

https://ebookcentral.proquest.com/lib/utasp/reader.action?docID=5190329\&ppg $=1$

Salazar, M. (2019). Experiencias y aprendizajes de la implementación de estrategias didácticas en educación virtual. Revista Científica Internacional, 1-9 https://www.researchgate.net/publication/344148220_Experiencias_y_aprendiza jes_de_la_implementacion_de_estrategias_didacticas_en_educacion_virtual

Salto, M. \& Cendón, A., (2019). Incidencia del Desempeño Profesional del Docente de Educación Inicial. Dialnet, 160-181.

Sampieri, R. H., Fernández Collado, C. \& Baptista Lucio, M. (2014). Metodología de la Investigación.

https://periodicooficial.jalisco.gob.mx/sites/periodicooficial.jalisco.gob.mx/files/ me todologia_de_la_investigacion_-_roberto_hernandez_sampieri.pdf

Szulkin, A. \& Correa, A. (2019). Una propuesta para el uso del teatro de títeres como herramienta socio-pedagógica en las escuelas rurales. Comunicarte.

Trueba, B. \& Rodríguez de la Flor, J. L. (2014). Títeres en el taller: el día a día de un taller de títeres con niños de 4 a 12 años. Barcelona: Ediciones Octaedro, S.L. https://elibro.net/es/ereader/uta/61965?as_all=e1\%20títere\%20\&as_all_op=unacc ent_icontains\&prev $=$ as\&page $=22$

Velazco, B. C. (2016). El Títere y su valor educativo. Análisis de su influencia en Titirimundi, Festival Internacional de Títeres de Segovia. https://dialnet.unirioja.es/servlet/tesis?codigo $=136515$

Wafunga,_ÂA. A., Rodríguez García, A. M. \& Fuentes Cabrera, A. (2018). Desenvolvimiento do pensamiento crítico reflexivo a través do ensino da história em Benguela. INNOEDUCA revista. https://revistas.uma.es/index.php/innoeduca/article/view/2523/4557

Zeinali, H. (2017). Los títeres como herramienta educativa. https://www.educacion.gob.es/teseo/imprimirFicheroTesis.do?idFichero=Qo1gw B Kv8ck 
El artículo que se publica es de exclusiva responsabilidad de los autores y no necesariamente reflejan el pensamiento de la Revista Conciencia Digital.

\section{Liencia}

El artículo queda en propiedad de la revista y, por tanto, su publicación parcial y/o total en otro medio tiene que ser autorizado por el director de la Revista Conciencia Digital.
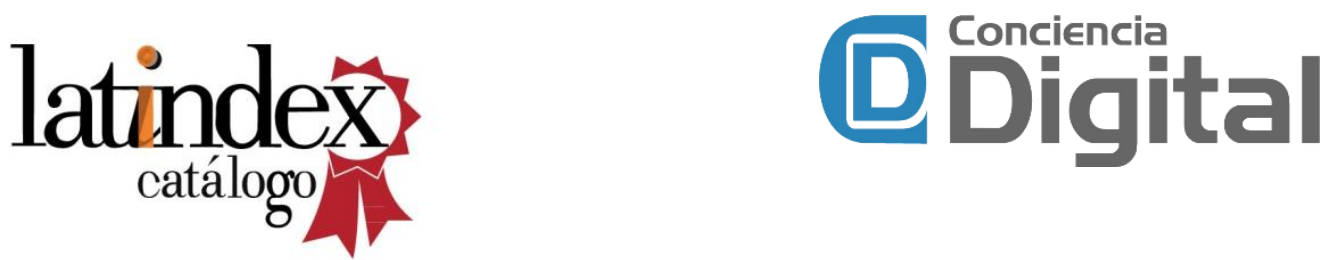

Indexaciones

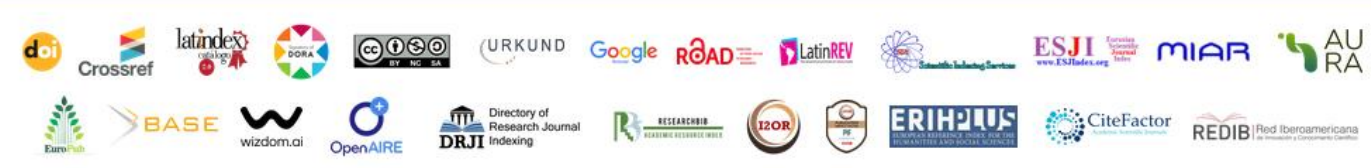

